\title{
Modified Small Incision Cataract Surgery (SICS) for combined extraction: A comparison of pre-operative and post-operative intraocular pressure (IOP)
}

\author{
Verma $\mathbf{A}^{1}$, Yadav $\mathbf{P}^{2}$ \\ ${ }^{1}$ Dr. Abha Verma, Associate Professor, ${ }^{2}$ Dr. Priyanka Yadav, Post Graduate; both authors are affiliated with Sri \\ Aurobindo Medical College and PG Institute, Indore, MP, India.
}

Corresponding Author: Dr. Priyanka Yadav, Post Graduate Resident, Sri Aurobindo Medical College and PG Institute, Indore, MP, India, E-mail: lifeisrace.11@gmail.com

\begin{abstract}
Aim: To find out surgical outcome in terms of IOP control, bleb anatomy and postoperative complications after combined surgery by using straight incision. Study design: Observational prospective study. Study centre: Conducted at tertiary health care centre. Material and methods: Study included 22 eyes of 20 patients with senile cataract and POAG (primary open angle glaucoma) who underwent SICS with trabeculectomy using straight incision were included in the study. Detailed anterior segment evaluation with IOP measurement was done on postoperative day one and then weekly for 6 weeks; thereafter at the end of 3rd, 6th and 12th month. Results: Preoperative mean IOP was $33.72 \pm 12.5$ $\mathrm{mm}$ of $\mathrm{Hg}$; after one year post-operative mean IOP was $12.41 \pm 3.6 \mathrm{~mm}$ of $\mathrm{Hg}$. Bleb was diffuse and flat comparatively. No major complications were seen. Conclusion: Straight incision for cataract extraction as well as trabeculectomy being easier for novice surgeon, with achievement of target IOP.
\end{abstract}

Keywords: Straight incision, Combined SICS, Trabeculectomy

\section{Introduction}

Glaucoma and cataract follow a silent and highly variable natural course and are the most common causes of visual handicap in senescence. Since 1960, trabeculectomy has been the most successful in lowering IOP in all types of glaucoma. First described by Cairns in 1968, with some modifications it is continued till date delivering variable results and efficacy [1]. Two basic principles for lowering IOP are: either increase outflow by dilating available anatomical route or making an artificial passage, or decrease secretion by medical or surgical means.

The INGOT randomized trial pioneered by Congdon et al, discovered better IOP lowering by trabeculectomy groups (36\% drop) than with medication groups $(23 \%$ drop). Where as regression model revealed $45.3 \%$ drop in IOP by trabeculectomy alone being superior to trabeculectomy using 5- fluorouracil combined with cataract surgery which brought a drop of $30.4 \%$, at one year follow up [2].

Manuscript received: $10^{\text {th }}$ November 2018

Reviewed: $20^{\text {th }}$ November 2018

Author Corrected: $28^{\text {th }}$ November 2018

Accepted for Publication: $1^{\text {st }}$ December 2018
Long term IOP is better controlled by combined cataract and glaucoma procedures than by cataract extraction alone [3]. Never theless, factors like extent of damage on diagnosis, compliance to medication, socioeconomic status and life expectancy of patient demands specifically tailored management.

With the use of antimetabolites, complications like over filtration resulted in hypotony with or without choroidal detachment, hypotonous maculopathy, insufficient drainage leading towards failure to reduce IOP, blebitis and endophthalmitis have been experienced [4]. Further, bleb related complications, large fluctuation of IOP, reduced efficacy lately and different learning curves were encountered with all the procedures [5]. With recent studies avoiding use of tissue antimetabolites, releasable sutures or laser suturelysis in conventional trabeculectomy, possibility to yield satisfactory control of IOP for many years has been stated $[6,7,8]$.

Treating patients with cataract and glaucoma by SICS and trabeculectomy (conventional or sutureless) has successfully fulfilled the aim of improving visual acuity 
and reducing IOP by means of one single procedure over past two decades [9]. Construction of uniform thickness scleral flap avoids button hole or thin and

\section{Material and Method}

Type of study- Prospective cross sectional study

Study centre- Tertiary health care center at Sri Aurobindo Medical College, Indore

Sampling method- patients were selected randomly who came to eye out patient department in between July 2016 to July 2018

Sample size- 22 eyes of 20 patients

Statistical Analysis-The data obtained were analyzed for statisticalsignificance using one way ANOVA.

\section{Inclusion criteria:}

1) Age $>50$ years and both gender.

2) Diagnosed as cataract with primary open/closed angle glaucoma.

3) Patients who gave consent for study.

\section{Exclusion criteria:}

1) Those with any other disorders (of cornea, vitreous and retina) and secondary glaucoma were excluded.

2) Patients who had history of uveitis, trauma, Neovascularization or past ocular surgery.

All cases were examined and operated by a single surgeon. Glaucoma was diagnosed after assessment of visual acuity with Snellen's chart, detailed slit lamp examination before and after dilatation, IOP measurement on applanation tonometer, fundoscopy, gonioscopy. Angle grading based on Scheie's system; to evaluate anatomical and functional status of each patient, cataract grading was done as per LOCS III (lens opacity classification system) [10,11]. All patients underwent SICS by using straight incision:

\section{Surgical Procedure[12, 13, 14]}

1. Surgery was performed under local peribulbar anaesthesia. Intraoperatively, IOP was maintained between 15 to $21 \mathrm{~mm}$ of $\mathrm{Hg}$, managed with intravenous fast infusion of $20 \%$ mannitol, if required.

2. A fornix based conjunctival flap was constructed, incising along the limbus between 10 to 2 o'clock, $6-8 \mathrm{~mm}$ in length and $4 \mathrm{~mm}$ in width.

3. After clearing Tenon's capsule and attaining haemostasis by wet field electrocautery, $6 \mathrm{~mm}$ long straight incision was made upto half the depth of scleral thickness, $2 \mathrm{~mm}$ away from limbus with No.15 Bard Parker knife. (fig.1)

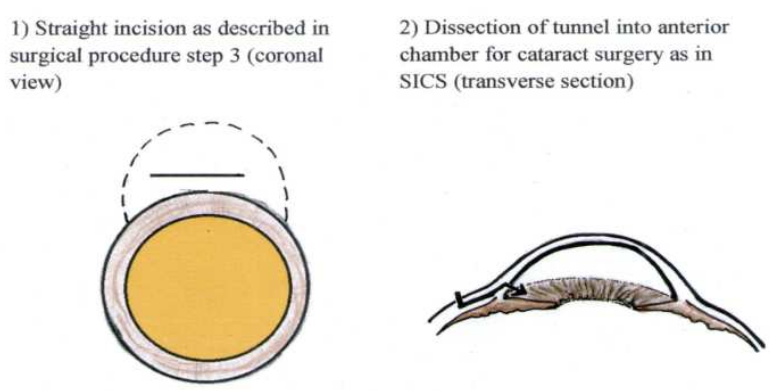

Figure 1: Diagrammatic picture showing straight incision site and tract

4. Sub scleral tunnel was made with crescent knife keeping side walls parallel, upto $2 \mathrm{~mm}$ of clear cornea without entering anterior chamber. 


\section{Original Research Article}

5. In contrast with SICS, length of scleral tunnel and inner end were equal in width without side pockets. An ophthalmologist trained in SICS has skill to modify the tunnel for sub-scleral trabeculectomy readily. (Figure.1)

6. After making a side port at 9 o' clock position standard steps of SICS were followed for ECCE and PCIOL implantation.

7. On visualizing limbal blue zone, a $2 \times 4 \mathrm{~mm}$ trabecular window was removed by the side port knife. Sharp vertical cuts provided non ragged ends of Schlemm's canal which opened in the scleral lake, formed by steps above. Thereafter this area was washed and swabbed. Scleral flap was sutured with interrupted 10-0 monofilament nylon radially at the edges, taking care that suture bite was full thickness in anterior lip and partially from scleral bed (Figure2)

8. Conjunctiva was reposited by an interrupted 7-0 vicryl sutures on each edge.

Results were evaluated in terms of IOP control, preservation of vision and any bleb related complications at day 1 followed up weekly till 6 weeks, further at the end of $3^{\text {rd }}, 6^{\text {th }}$ and $12^{\text {th }}$ month, post operatively. Success rate was defined as post operative IOP of 10- $18 \mathrm{~mm} \mathrm{Hg}$ without medication for period of 6 months, without any major complications [15].

\section{Results}

In our study we included patients who aged $>50$ years, the mean age was 64.5 years. Out of 22 patients $12(54.5 \%)$ were male and $10(45.5 \%)$ patients were female (Table.1)

Right eye was operated of $14(63.6 \%)$ patients and left eye in $8(36.3 \%)$ patients. (Table.1)

On the basis of type of glaucoma 6 (27.2\%) had open angle and 16 (72.7\%) patients had Closed angle on gonioscopy. Grading of cataract was done according to LOCSIII $^{9}$, in our study: 3 (13.6\%) had NS I, 16 (72.7\%) had NS II and 3 (13.6\%) had NS III. (Table.1)

Table-1: Demographic profile of the patients.

\begin{tabular}{|l|c|}
\hline Age mean in years & $\mathbf{6 4 . 5}$ years \\
\hline Sex & \\
$\bullet$ Men & $12(54.5 \%)$ \\
& $10(45.5 \%)$ \\
\hline $\begin{array}{l}\text { Laterality } \\
\bullet \text { Right }\end{array}$ & $14(63.6 \%)$ \\
$\bullet$ Left & $8(36.3 \%)$ \\
\hline Type of glaucoma & \\
$\bullet$ Open angle & $6(27.2 \%)$ \\
$\bullet$ Closed angle & $16(72.7 \%)$ \\
\hline Grade of cataract (LOCSIII) \\
$\bullet$ NS I & $3(13.6 \%)$ \\
$\bullet$ NS II & $16(72.7 \%)$ \\
$\bullet$ NS III & $3(13.6 \%)$ \\
\hline
\end{tabular}

Table-2: Preoperative and postoperative IOP comparison.

\begin{tabular}{|c|c|}
\hline Mean IOP & IOP $(\mathbf{m m}$ of $\mathbf{H g})$ \\
\hline Preoperative & $33.72 \pm 12.5$ \\
\hline Postoperative & $12.41 \pm 3.6$ \\
\hline
\end{tabular}

For analyzing the efficacy of procedures extent of IOP lowering was noted. The procedure showed lowering of IOP with the mean difference in IOP of $21.31 \mathrm{~mm}$ of $\mathrm{Hg}$ (Figure. 3). Preoperative mean IOP was $33.72 \pm 12.5 \mathrm{~mm}$ of $\mathrm{Hg}$; after one year post-operative mean IOP was $12.41 \pm 3.6 \mathrm{~mm}$ of $\mathrm{Hg}$ (Table. 2). The most common complication was hypotony which was noted on 1 st postoperative day in $16(72.7 \%)$ patients (Table 3 ) 
Table-3: Postoperative complications.

\begin{tabular}{|c|c|c|}
\hline Complications at $\mathbf{1}^{\text {st }}$ postoperative day & No. of patients & Percentage \\
\hline Hyphaema & 1 & $4.5 \%$ \\
\hline Hypotony & 16 & $72.7 \%$ \\
\hline PCR & 1 & $4.5 \%$ \\
\hline Hypertony & 1 & $4.5 \%$ \\
\hline Shallow anterior chamber & 4 & $18.1 \%$ \\
\hline
\end{tabular}

\section{Discussion}

In the Indian scenario, due to continuing financial constraints or refractory nature of the disease to medical therapy with time, there is a limitation on part of the patients to follow medical management. In such tough call, combined extraction has been a more efficacious alternative of controlling IOP [12].

In our study 22 eyes were evaluated to ensure the efficacy of Modified Small Incision Cataract Surgery (SICS) For Combined Extraction. Sample composed of $12(54.5 \%)$ male and 10(45.5\%) female. Mean age of presentation was 64.5 years.

Majority of cases were presented with closed angle glaucoma 16 patients and 6 cases were presented with open angle glaucoma. All patients with nucleus sclerosis were included.

Preoperatively the mean IOP was $33.72 \pm 12.5 \mathrm{~mm}$ of $\mathrm{Hg}$ and postoperatively it was $12.41 \pm 3.6 \mathrm{~mm}$ of $\mathrm{Hg}$. Patients exhibited mean reduction in IOP of $21.31 \mathrm{~mm}$ of $\mathrm{Hg}$ post-operatively, after one year follow up with diffuse functional bleb.

In all our cases bleb was diffuse but target IOP was maintained throughout the period of one year. As compared to conventional trabeculectomy aqueous leak is more in straight incision modified combined SICS so post operatively diffuse bleb with shallow AC was noted $[15,16]$. In $4(18 \%)$ patients shallow anterior chamber was found with a negative Siedel's test but releasable sutures were avoided.

Study by Vasanthi et al noted $11.4 \pm 3.1 \mathrm{mmHg}$ mean intraocular pressure (IOP) on the first postoperative day in the tunnel incision group $(\mathrm{P}=0.012)$ with diffuse bleb. Instead of raised bleb diffuse bleb was noted may be due to large incision size (6-7 mm) comparatively [17].

Intraoperatively in only 1 case PCR was encountered except that no other complications were encountered while performing surgery.
On first postoperative day we noted Hyphaema in $4.5 \%$, Hypotony in $72.7 \%$, Hypertony in $4.5 \%$ and Shallow anterior chamber in $18.1 \%$ cases. Cases with shallow $\mathrm{AC}$ and hypotony owing to fruitful conservative alternative; achieved by tight patching with $1 \%$ atropine sulphate ointment overnight. No major complications were encountered. Hyphaema was resolved within a week with continuation of routine medication postoperatively.

In a study by Usha B. R. et al similar postoperative complications Shallow AC andover-filtration in $8 \%$, Fibrinousreaction in AC 14\%, choroidal detachment $4 \%$ and hyphaema in $16 \%$ cases were noted and were managed successfully with medical treatment [14].

We preferred limbal based conjunctival flap which also helps in maintaining IOP post operatively. Precise operative technique plays a major role in achieving success. IOP reduction with a limbus basedconjunctival flap is supposed to be more helpful than with a fornix based flap [18].

In order to cause lesser surgically induced astigmatism, scleral wound was radially sutured resulting in better healing with a diffuse but functional bleb. Considering patients poor hygienic dwelling, this modification not only channelized required outflow of aqueous but also protected patients from endophthalmitis.

A study done at Nepalmentions complications related to laser suturelysis (which requires costly equipment) like bleb leak and blebitis; early releasable sutures may have higher incidence of hypotonyand bleb failure [19].

No bleb related complications were noted after one year follow up, no antimetabolite was used in our study [20].

\section{Conclusion}

The silently blinding Indian society burdened with the enormous backlog suffering from cataract and glaucoma 


\section{Original Research Article}

awaiting a timely rescue outnumbers country's experienced surgeons. To join this battle against the sight threatening duo, novice surgeons can play their part, being familiar and at ease with straight incision SICS, safely but economically as well. The combined SICS via straight incision with IOL implantation and trabeculectomy is a boon to deliver fairly satisfactory outcomes. After glaucoma surgery worsening of cataract iseliminated. Intraocular pressure (IOP) control is usually better after acombined procedure than after cataract surgery [17]. In our study combined surgery was performed by single surgeon (unit head) with preoperative and postoperative examination. Data collection and manuscript work was done by resident under consultant supervision and guidance.

What we learn from this study-In our study with a reasonable visual restoration, satisfactory IOP control was achieved without any IOP lowering drugs, post operatively.

Patients in developing countries who are not able to afford antiglaucoma medication are at risk for further progression of disease; their sight could be saved by this combined approach to reduce the burden of blindness.

Combined approach to cataract and glaucoma is an option of choice for patients with poor compliance or patients who are not on regular follow ups.

In our study IOP was noted within normal range despite of diffuse flat bleb.

\section{Funding: Nil, Conflict of interest: Nil Permission from IRB: Yes}

\section{References}

1. Cairns JE. Trabeculectomy. Preliminary report of a new method. Am J Ophthalmol. 1968 Oct;66(4):673-9.

2. Congdon NG, Krishnadas R, Friedman DS, et al. A study of initial therapy for glaucoma in southern India: India Glaucoma Outcomes and Treatment (INGOT) Study. Ophthalmic Epidemiol. 2012 Jun; 19 (3): 14958. doi: 10.3109/09286586.2012.667493.

3. Friedman DS, Jampel HD, Lubomski LH, et al. Surgical strategies for coexisting glaucoma and cataract: an evidence-based update. Ophthalmology. 2002 Oct; 109 (10):1902-13.

4. Vesti E, Raitta C. A review of the outcome of trabeculectomy in open-angle glaucoma. Ophthalmic Surg Lasers. 1997 Feb;28(2):128-32.
5. Solus JF, Jampel HD, Tracey PA, et al. Comparison of limbus-based and fornix-based trabeculectomy: success, bleb-related complications, and bleb morphology. Ophthalmology. 2012 Apr; 119 (4): 70311. doi:10.1016/j.ophtha.2011.09.046. Epub 2012 Jan 9.

6. D'Ermo F, Bonomi L, Doro D. A critical analysis of the long-term results of trabeculectomy. Am J Ophthalmol. 1979 Nov; 88 (5):829-35.

7. Mills KB. Trabeculectomy: a retrospective long-term follow-up of 444 cases. Br J Ophthalmol. 1981 Nov; 65 (11):790-5.

8. Nouri-Mahdavi K, Brigatti L, Weitzman M, Caprioli J. Outcomes of trabeculectomy for primary open-angle glaucoma. Ophthalmology. 1995 Dec;102(12):1760-9.

9. Gayton JL, Van Der Karr M, Sanders V. Combined cataract and glaucoma surgery: trabeculectomy versus endoscopic laser cycloablation. J Cataract Refract Surg. 1999 Sep;25(9):1214-9.

10. Karbassi M, Khu PM, Singer DM, Chylack LT Jr. Evaluation of lens opacities classification system III applied at the slitlamp. Optom Vis Sci. 1993 Nov; 70 (11):923-8.

11. Chylack LT Jr, Wolfe JK, Singer DM, et al. The Lens Opacities Classification System III. The Longitudinal Study of Cataract Study Group. Arch Ophthalmol. 1993 Jun;111(6):831-6.

12. Singh K, Mutreja A, Jain P. A Comparative Evaluation of Phacotrabeculectomy with Manual Phacofracture Trabeculectomy. MAMC J Med Sci 2015; 1: 6-11.

13. Natchiar G, Dabralkar T. Manual small incision sutureless cataract surgery- an alternative technique to instrumental phacoemulsification.Operative Techniques Cataract Refrat Surg. 2000; 3: 161- 70.

14.Usha B.R., Usha M., Prasad B. Outcome of conventional trabeculectomy with or without cataract surgery. Int J Cur Res Rev September 2015; 7:17, 2026.

15. Narita A1, Morizane Y2, Miyake T3, Seguchi J3, Baba T4, Shiraga F2. Characteristics of successful filtering blebs at 1 year after trabeculectomy using swept-source three-dimensional anterior segment optical coherence tomography. Jpn J Ophthalmol. 2017 May; 61 (3): 253-259. doi: 10.1007/s10384-017-0504-2. Epub 2017 Feb 14. 


\section{Original Research Article}

16. Rossmann M, Harrer S, Rigal K. T-cut in the bottom of the scleral pocket in combined cataract and glaucoma surgery. J Cataract Refract Surg. 2000 May;26(5):702-8

17. Kotian VB, Shetty J, Pai V, Kulkarni U, Bhat S, Shetty H, Bangera A. Manual small incision cataract surgery within the tunnel, trabeculectomy in patients having primary open angle glaucoma and cataract. Guoji YankeZazhi (Int Eye Sci) 2012;12 (9):1609-1613.

18. Sandra J. Sofinski, Pranav Amin, and R. R and Allingham, Chapter 226: Glaucoma filtration surgery. In Principles and Practice of Ophthalmology, Albert and
Jakobiec, Azar, Gragoudas; volume 4, 2nd ed. W.B. Saunder company; 2000. pp 2959-60.

19. Kumari R., Badhu BP, Das H. Effectiveness of combination of permanent and releasable scleral flap sutures in trabeculectomy: A randomized clinical trial. Kathmandu University Medical Journal 2006 Vol.4; No.4, Issue 16: 419 - 425.

20. Prasad VN, Narain M, Bist HK, Khan MM. Trepano-trabeculectomy (a combined operation for glaucoma). Indian J Ophthalmol. 1984 Mar-Apr; 32 (2): $73-5$.

\section{How to cite this article?}

Verma A, Yadav P. Modified Small Incision Cataract Surgery (SICS) for combined extraction: A comparison of preoperative and post-operative intraocular pressure (IOP). Trop J Ophthalmol Otolaryngol.2018;3(4):63-68.doi: 10.17511/jooo.2018.i04.01 